\title{
Intraventricular Central Neurocytoma
}

\author{
M. Giroux, J.P. Farmer, K.M. Meagher-Villemure, A.M. O’Gorman and J.L. Montes
}

\begin{abstract}
A case of central neurocytoma treated surgically is described. The authors review the literature. Emphasis is placed on radiological and pathological features not previously described. In particular, the intra-operative ultrasound appearance is described. The role of adjunctive radiotherapy is also discussed.
\end{abstract}

RÉSUMÉ: Nous présentons un cas de neurocytome central traité chirurgicalement ainsi qu'une revue de littérature. Une attentiion particulière est dévouée aux caractéristiques radiologiques et pathologiques uniques de cette lésion. L'apparence de l'ultrason peropératoire, jamais décrite auparavant, est élaborée. Le plan thérapeutique postopératoire ainsi que le rôle de la radiothérapie d'appoint pour le traitement de ces lésions sont discutés.

Can. J. Neurol. Sci. 1992; 19: 392-396

We report the clinical presentation, the radiological findings, and the pathologic features of a patient with an intraventricular neurocytoma successfully resected via a transfrontal approach, and we review the literature on this rare entity. ${ }^{\prime}$ Particular emphasis is placed on previously unreported radiological, surgical and pathological features of these tumors which may help distinguish neurocytoma from other intraventricular tumors, and therefore, facilitate diagnosis.

\section{CASE Report}

This patient is a 17-year-old right-handed female who presented with a three week history of daily bifrontal throbbing headaches, nausea and vomiting. The headaches woke her up once at night, but usually were worse in the morning. She also complained of bilateral tinnitus, worse on the right side, photophobia, blurred vision, and intermittent diplopia. She also noted urinary frequency. The physical examination showed bilateral papilloedema and left-sided hyperreflexia. There were no abnormal cutaneous features.

\section{Radiological findings}

A CT scan of the brain showed a hyperdense, somewhat speckled mass, suggestive of fine calcifications in the right lateral ventricle associated with ventriculomegaly. The mass enhanced inhomogeneously after contrast injection (Figure 1).

Because very vascular tumors such as choroid plexus papilloma and carcinoma were part of the differential diagnosis, cerebral angiography was done. It revealed a normal arterial phase except for elevation of pericallosal arteries secondary to ventriculomegaly, but showed a vascular blush in the region of the right lateral ventricle persisting into the late venous phase (Figure 2).

The MRI in spin echo technique showed a slightly hyperintense mass within the body of the right lateral ventricle on $\mathrm{Tl}$ weighed images. Following gadolinium injection, there was a moderate inhomogeneous enhancement of the ventricular mass (Figure 3). The mass filled the portion of the ventricle above the right thalamus, displacing the interventricular septum to the left side and obstructing both foramina of Monro. Its inferior margin was at the roof of the 3 rd ventricle. There was bilateral, lateral ventricular dilatation, but the $3 \mathrm{rd}$ and $4 \mathrm{th}$ ventricles were within normal limits in size.

\section{Management}

A left external ventricular drain was inserted with relief of headaches but the diplopia persisted. The patient underwent a right frontal craniotomy. Because of the massive right frontal horn dilatation, and because of the size of the tumor, a transcortical approach through the second frontal gyrus was favored over a transcallosal approach. Intra-operative ultrasound was used to localize the tumor and the frontal horn which was initially punctured with a brain needle. The needle tract was then followed and the ventricular window widened.

A soft, violaceous, red, and quite hemorrhagic tumor that appeared adjacent to, but free of the ependymal roof as well as of the lateral ventricular wall was found. It had caused chronic changes in the septum pellucidum which was discolored yellow. The tumor extended towards the foramina of Monro and was attached to the choroid plexus along the choroidal fissure. The tumor was resected by ultrasonic aspiration. At the end of the resection, a one centimetre fenestration was made in the septum pellucidum to avoid sequestered hydrocephalus.

There was no post-operative complication and five days later, a permanent left ventriculo-peritoneal shunt was inserted when the patient was noted to be shunt dependent.

The patient made an uneventful recovery except for one seizure secondary to sub-therapeutic levels of phenytoin and she was discharged home one week after the shunt insertion. Post-operative

From the Departments of Neurosurgery (M.G., J.P.F., J.L.M.), Pathology (K.M.M.-V.) and Radiology (A.M.O.), The Montreal Children's Hospital, McGill University, Montreal

Received December 30, 1991. Accepted in final form April 3, 1992

Reprint requests to: J.P. Farmer, M.D., Division of Neurosurgery, The Montreal Children's Hospital, 2300 Tupper Street Rm C-811, Montreal, Quebec, Canada H3H 1 P3 


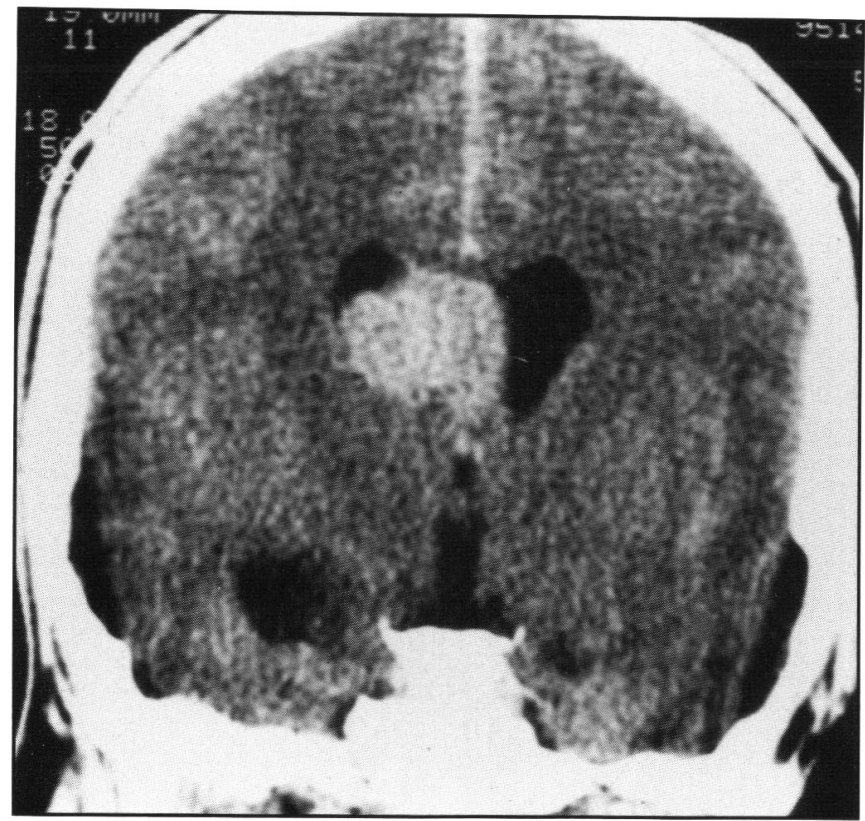

A

Figure I - CT appearance of neurocytoma. (1A) Coronal CT without contrast. Note speckled hyperdensity, bowing of septum and hydrocephalus. (IB) Axial CT with contrast injection. Note minimal and irregular enhancement.

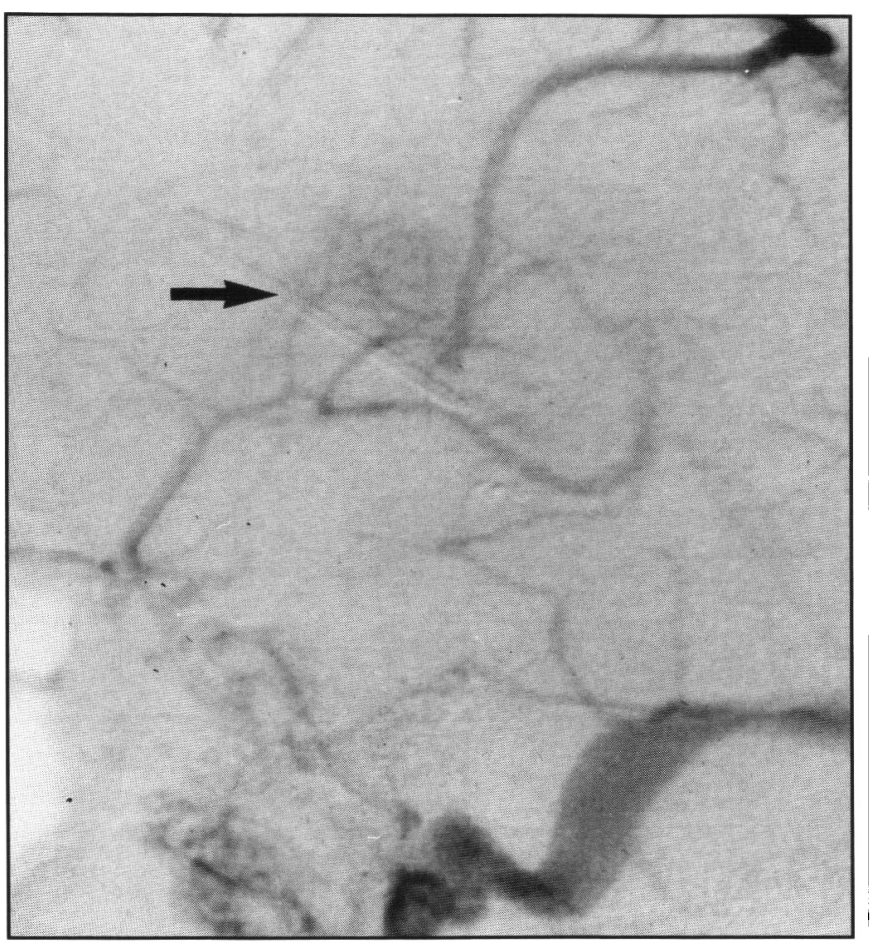

Figure 2 - Lateral Digital Substraction Angiogram. Note persistent faint blush during venous phase in region of caudate and thalamostriate veins (arrow).

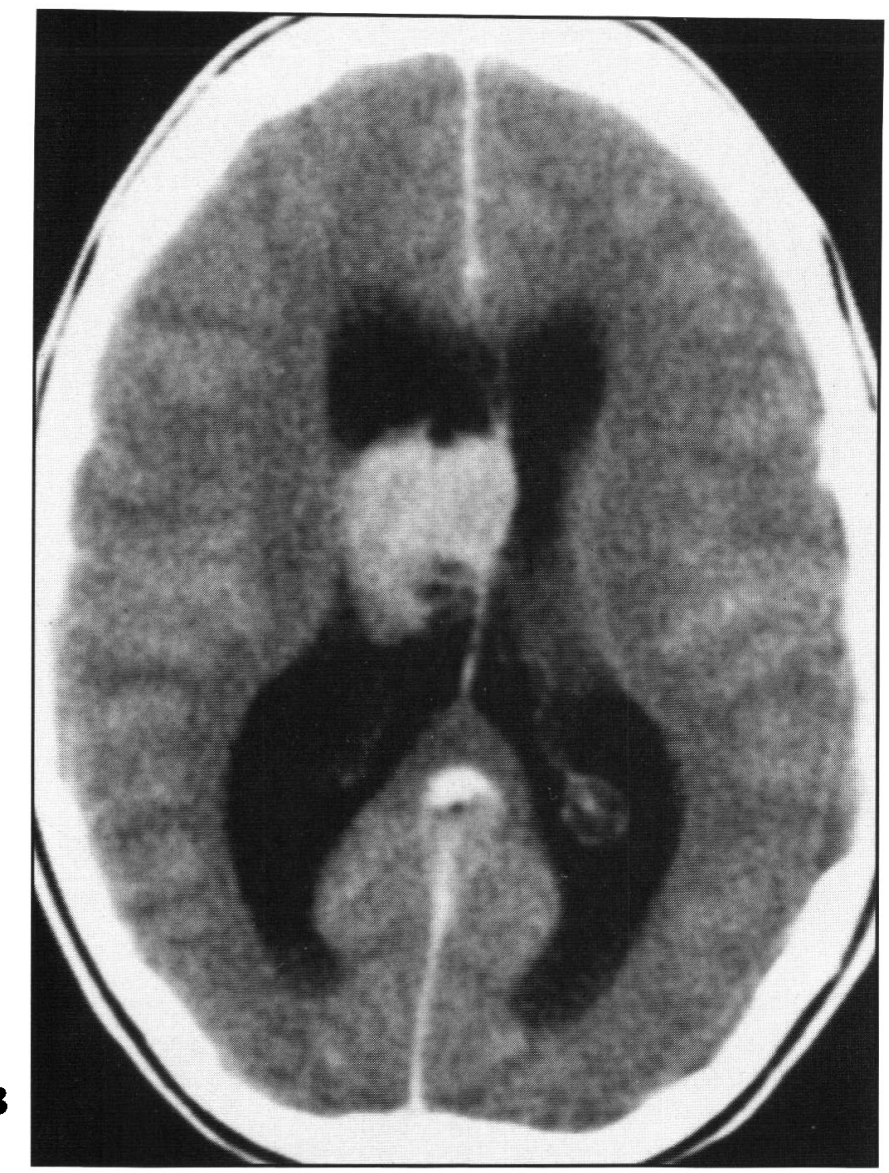

CT scan, and MRI confirmed complete resection of the tumor (Figure 4). Twelve months later, at follow-up visit, she was asymptomatic and seizure-free, on Tegretol therapy. Adjuvant therapy was not given.

\section{Pathological findings}

The light microscopy consisted of a diffuse proliferation of uniform appearing cells having a small, round nucleus in a small, eosinophilic or clear cytoplasm. Some cells had a rosette arrangement with thin fibrillary processes in their centres, and others were distributed around vessel-like papillary structures or pseudorosette arrangements. There were numerous sites of early hemorrhages within this richly vascularized tumor. The light microscopy findings were not dissimilar to that of oligodendroglioma in which perinuclear halos can be found or to that of ependymoma in which pseudorosettes are seen (Figure 5).

The electron microscopy showed tumor cells with small, round nuclei in a small cytoplasm containing multiple processes. Rare microfilaments were seen in these cytoplasms as well as many mitochondriae with bizarre cristae, numerous microtubules, many electron-dense granules, definite neurosecretory granule formations and some clear vesicles (Figure 6). Although intercellular junctions were seen, no synaptic complexes were found.

Immunohistochemistry was done to search for particular features in these tumor cells. The GFAP stain was focally positive in foot processes around some blood vessels but not in the tumor cells (corresponding to reactive astrocytes). The N.S.E. (neurone specific enolase) was positive in almost every tumor 


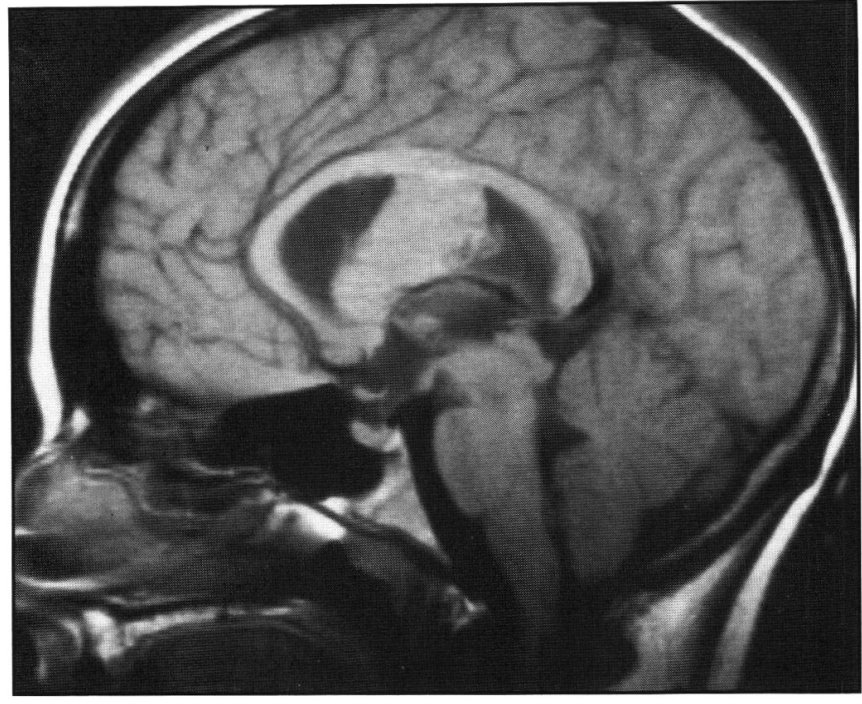

A

Figure 3-MRI appearance of neurocytoma. (3A) Sagittal appearance of tumor. Note close relationship with foramina of Monro and posterior extension in the ventricle. (3B) Coronal MRI post Gadolinium revealing a heterogeneous enhancement and bowing of septum pellucidum.

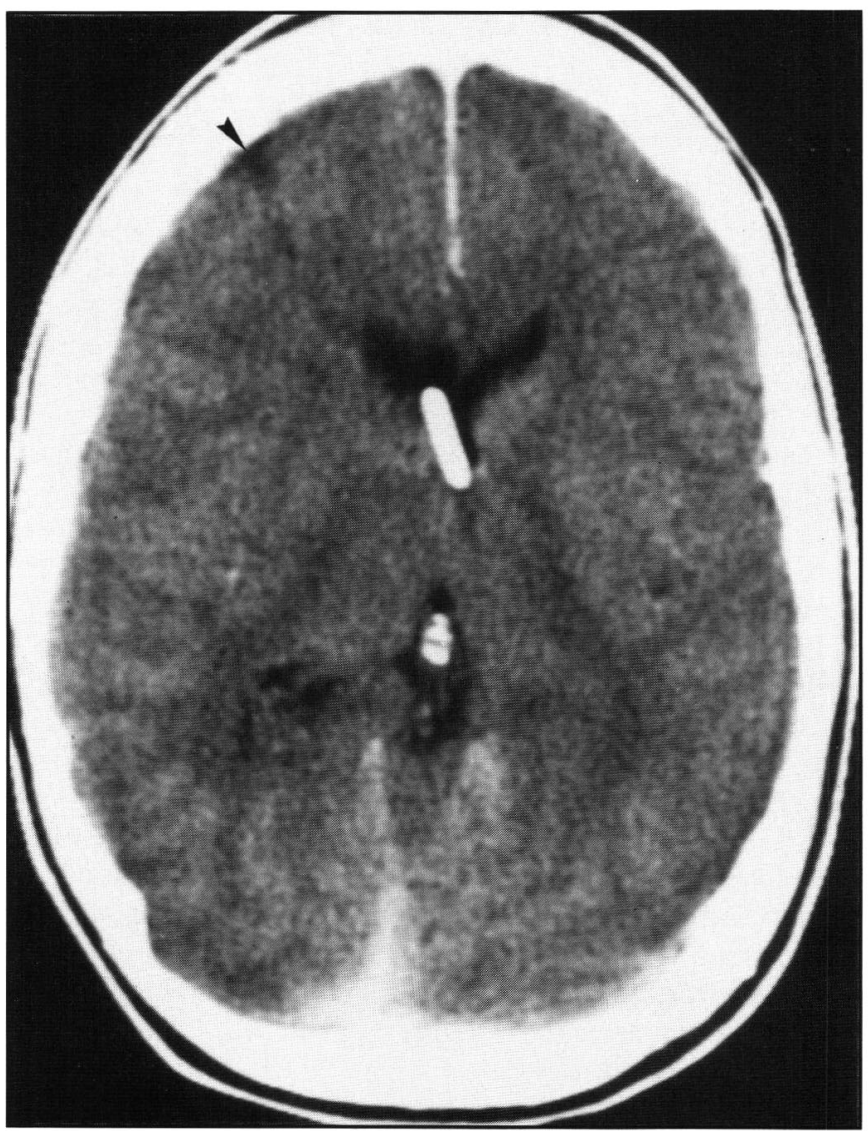

Figure 4-Post-operative CT with contrast. Note resolution of hydrocephalus, left V.P. shunt residual deformity of the right frontal horn and surgical tract through frontal lobe (arrowhead).

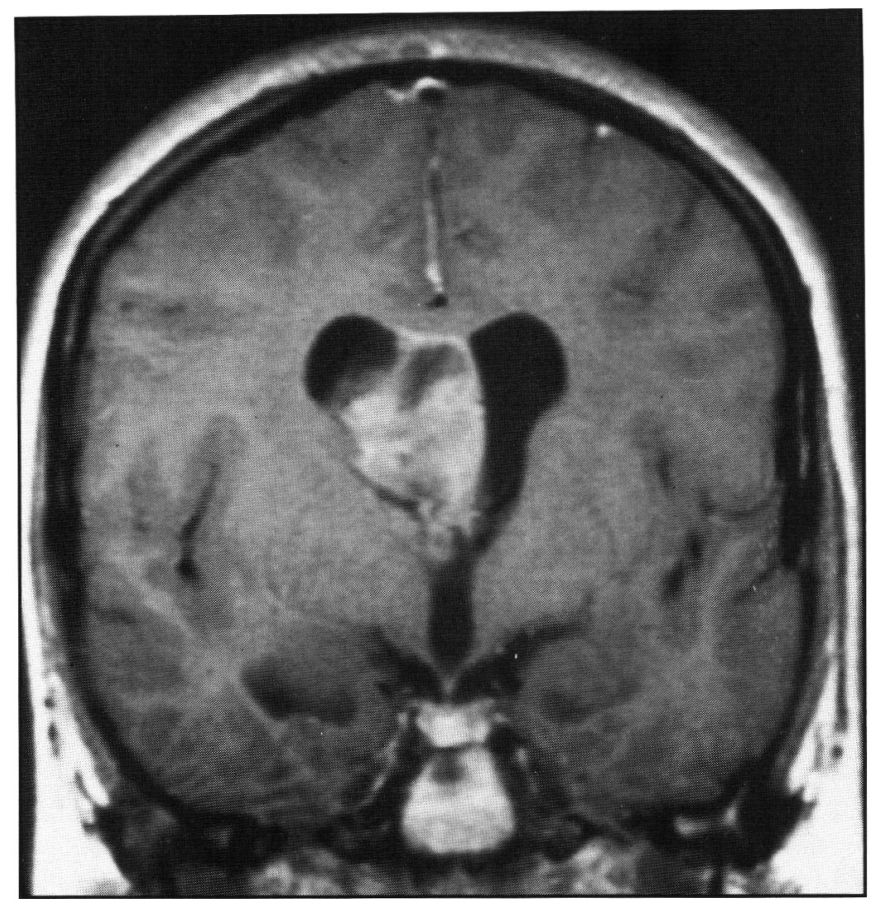

B

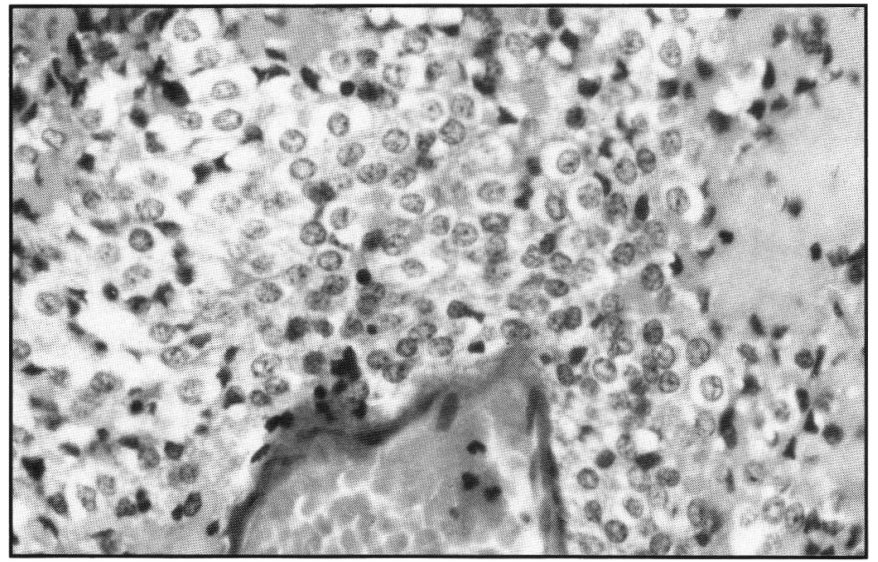

Figure 5 - Area of tumor demonstrating oligodendroglioma-like architecture with recent hemorrhage. HPS $\times 400$.

cell cytoplasm. The synaptophysin was occasionally positive in some large cells' cytoplasm, revealing the presence of portions of synapse complexes.

The presence of neurotubules, neurosecretory granules, clear vesicles and well-developed intercellular junctions on ultrastructure and the positive immunostaining for N.S.E. and synaptophysin suggested a neuronal origin of the tumor, consistent with a neurocytoma.

\section{Discussion}

Intraventricular tumors derived from neuronal cells are rare and have been divided into neuroblastoma and more differentiated neurocytoma. ${ }^{2}$ Neurocytomas occur more frequently in young adults ${ }^{3-8}$ with no sex predominance. There is a greater 


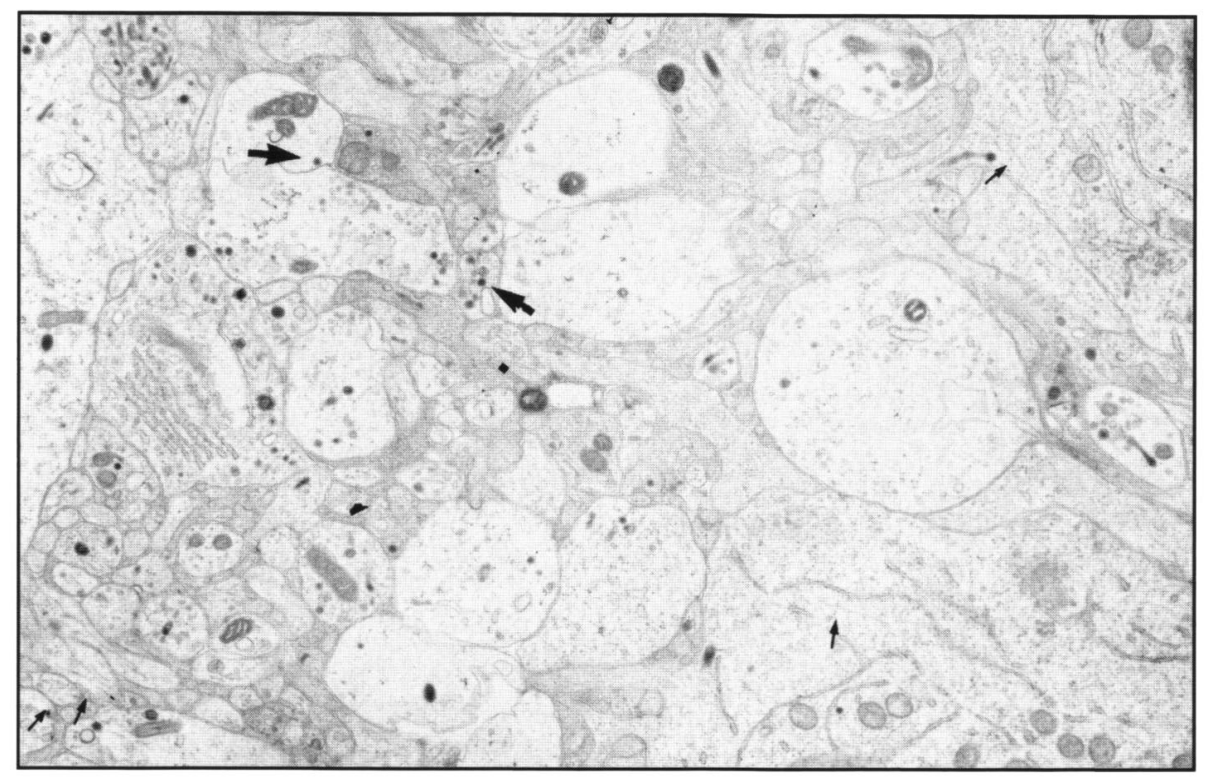

Figure 6 - Ultrastructure of the tumor cell processes in which neurosecretory granules (large arrows) and microtubules are easily found $x$ 10, 760 (small arrows).

incidence of these neoplasms in the lateral ventricles ${ }^{1-3.6 .9 .10}$ and they can occasionally reach the third ventricle, obstructing the foramina of Monro to produce lateral ventricular dilatation. Usually, patients present with signs of increased intracranial pressure secondary to acute hydrocephalus. Cases presenting as sudden death ${ }^{2}$ and frontal syndrome ${ }^{2}$ have been reported in the literature. In the majority of these patients, no localizing neurological signs can be found on physical examination..$^{2-4}$

Cerebral CT scan usually shows an intraventricular partly calcified enhancing mass associated with dilatation of one or both lateral ventricles. ${ }^{2-4,9}$ Cerebral arteriography fails to reveal any increase in vascularity in these masses. ${ }^{2.4 .9}$ With intraventricular masses, papilloma, meningioma, ependymoma and oligodendroglioma must be included in the radiological differential diagnosis.

Electron microscopic features and immunoperoxidase investigations are needed to differentiate neoplasms of neuronal cell origin from the other lesions. The abundant cell processes containing parallel microtubules, neurosecretory granules, clear vesicles and well developed intercellular junctions seen at electron microscopy clearly indicate the neuroblastic nature of the tumor. $3.7,9.11$ The presence of more mature neuronal tissue with defined synapses and synaptic junctions as seen with immunostaining has been one of the critical features distinguishing neurocytoma from differentiated neuroblastoma. ${ }^{1-3,9,11}$

Our case is similar to others found in the literature..$^{-3,6,9,10}$ The patient is a young adult who presented with signs of increased intracranial pressure. The CT scan appearance and the location of the mass are typical, but the arteriography revealed tumor hypervascularity with vascular blush persisting in the venous phase; a phenomenon not usually described. This preoperative feature correlated well with the surgical finding of a particularly hemorrhagic tumor.

MRI showed an intraventricular hyperintense mass on T1 weighed images which enhanced inhomogeneously after gadolinium injection. Ultrasound showed a hetero-echogenic mass and this feature facilitated surgical localization. The echogenecity was not dissimilar to that of ependymoma. The latter tends to be more uniform, however, unless the tumor has been previously irradiated. To our knowledge, ultrasound appearance of the neurocytoma has not previously been described.

The light microscopic features of the tumor were not specific and quite reminiscent of an oligodendroglioma or an ependymoma, but the electron microscopic findings suggested a neuronal tumor. Even if no synapses were seen at EM, the true neuronal nature of the mass was suggested by the marked neurone specific enolase (NSE) positivity. Synaptophysin revealed the presence of true synapses and confirmed the more differentiated nature of this neuronal tumor pointing towards a neurocytoma. Considering the location, the age of the patient, the immunohistochemistry, the E.M. features, as well as with N.S.E. and synaptophysin stains, the diagnosis of central neurocytoma was confirmed.

The exact origin of this tumor is unknown but Hassoun et al. ${ }^{2}$ suggested the neuronal cells of the septum pellucidum. Zimmerman proposed that it could arise from a sub-ependymal remnant of neuroectodermal origin growing in the third ventricle area. ${ }^{7}$ Our surgical findings of septum pellucidum discoloration could support the first hypothesis, but could also represent a non-specific reaction of septal cells to chronic pressure or minor remote hemorrhage. Once the bulk of the tumor was removed, residual cells were difficult to distinguish from the choroid plexus along the fissure. Both cell types are violaceous and can be hemorrhagic, but the infrastructure of choroidal cells is firmer and less amenable to microsuction. We would feel more strongly, based on our surgical findings, that the point of attachment and, therefore, of origin of this tumor might be the choroidal fissure.

There is a general agreement in the literature that complete surgical removal of the tumor is the treatment of choice. ${ }^{1-3.6 .9}$ It 
is not clear, however, if these patients should be irradiated after the surgery. Nishio et al. ${ }^{4}$ mention a patient surviving well over eighteen years following incomplete resection with irradiation. Yazargil et al. ${ }^{12}$ report a survival of twelve years after complete surgical excision only. He also reports a recurrence (histology unchanged) three years after complete excision and the presence of subtypes of neurocytomas with more aggressive pathological features which have been irradiated. Our patient will be followed by serial neurological and radiological examinations. Consideration of irradiation would be given at the first sign of recurrence.

\section{REFERENCES}

1. Buerger PC, Scheithauer BW, Vogel FS. Surfical pathology of the nervous system and its coverings. Churchill-Livingstone 1991: 324-325.

2. Townsend JJ, Seaman JP. Central neurocytoma, a rare benign intraventricular tumor. Acta Neuropathol (Berl) 1986; 71: 167-170.

3. Hassoun J, Gambarelli D, Griseli F, et al. Central neurocytoma. An electron microscopic study of two cases. Acta Neuropathol (Berl) 1982; 56: 151-156.

4. Nishio S, Tashima T, Takeshita I, et al. Intraventricular neurocytoma: clinicopathological features of six cases. J Neurosurg 1988; 68: 665-670.
5. Pearl GS, Takei Y, Bakay RA, et al. Intraventricular primary cerebral neuroblastoma in adults: report of three cases. Neurosurgery $1985 ; 16: 847-849$.

6. Pearl GS, Takei Y, Stefanis GS, et al. Intraventricular neuroblastoma in a patient with Von Hippel-Lindau's disease. Light and electron microscopic study. Acta Neuropathol (Berl) 1981; 53: 253-256.

7. Poon TP, Mangiardi JR, Matosu I, et al. Third ventricular primary cerebral neuroblastoma. Surg Neurol 1988; 30: 237-241.

8. Silver JM, Rawlings CE, Rossitol E. Ganglioglioma: a clinical study with long term follow up. Surg Neurol 1991; 35: 261-266.

9. Wichmann W, Schubiger O, Primling AV, et al. Neuroradiology of central neurocytoma. Neuroradiology 1991; 33: 143-148.

10. Wilson AJ, Leafter DH, Kohout ND. Differentiated cerebral neuroblastoma: a tumor in need of discovery. Hum Pathol 1988; 16 : 647-649.

11. Langford LA, Camel MH. Palisading pattern in cerebral neuroblastoma mimicking the primitive polar spongio-blastoma - an ultrastructural study. Acta Neuropathol (Berl) 1987; 73: 153159.

12. Yasargil GM, Von Ammon K, Von Deim Ling A, et al. Central neurocytoma: histo-pathological variants and therapeutic approaches. J Neurosurg 1992; 76: 32-37. 